\title{
Impact of tidal Poisson terms on nonrigid Earth rotation
}

\author{
M. Folgueira ${ }^{1,2}$, V. Dehant ${ }^{3}$, S. B. Lambert ${ }^{3}$, and N. Rambaux ${ }^{3,4}$ \\ 1 Instituto de Astronomía y Geodesia (UCM-CSIC), Facultad de Ciencias Matemáticas, Universidad Complutense de Madrid, \\ 28040 Madrid, Spain \\ e-mail: martafl@mat.ucm.es \\ 2 Observatoire de Paris, SYRTE, CNRS/UMR8630, 75014 Paris, France \\ 3 Royal Observatory of Belgium, 1180 Brussels, Belgium \\ e-mail: v.dehant@oma.be,s.lambert@oma.be \\ 4 Depart. of Mathematics, Facultés Univ. N. D. de la Paix, 5000 Namur, Belgium \\ e-mail: Nicolas.Rambaux@fundp.ac.be
}

Received 27 November 2006 / Accepted 3 April 2007

\begin{abstract}
Context. The tidal potential generated by bodies in the solar system contains Poisson terms, i.e., periodic terms with linearly timedependent amplitudes. The influence of these terms on the Earth's rotation, although expected to be small, is of interest for high accuracy modeling.

Aims. Therefore, we study their contribution to the rotation of a non-rigid Earth with an elastic mantle and liquid core.

Methods. Starting from Liouville's equations, and following an analytical treatment, we obtain the relations accounting for Poisson terms in the forcing and providing the solution for the wobble.

Results. We show that the transfer function between rigid and non rigid nutation amplitudes, as usually defined in the literature, must be supplemented by additional terms proportional to the amplitude of the Poisson term of the potential. These new terms are inversely proportional to $\left(\sigma-\sigma_{\mathrm{N}}\right)^{2}$ where $\sigma$ is the forcing frequency and $\sigma_{\mathrm{N}}$ are the eigenfrequencies associated with the retrograde free core nutation and the Chandler wobble. The highest contribution to the nutation is $6 \mu$ as $(\Delta \psi)$ on the term $2 l^{\prime}-2 F+2 D-2 \Omega$ and remains below $1 \mu$ as for the other terms. A contribution of $88 \mu \mathrm{as} / \mathrm{cy}$ is found to the obliquity rate. We evaluate the variations of the third component of the wobble of the Earth and of the core in response to a zonal tidal potential, and show that there is no significant change.
\end{abstract}

Key words. reference systems - Earth

\section{Introduction}

The precession and nutation of the Earth are usually computed from a rigid Earth nutation theory combined with a transfer function accounting for the non-rigid Earth contribution. The International Astronomical Union (IAU) has recommended the use of the IAU 2000A nutation model, based on the work of Mathews et al. (2002), from 1 January 2003. This model took advantage of recent advances in rigid and non-rigid Earth theoretical modelling as well as developments in very long baseline radio interferometry (VLBI) instrumental, observational and analysis strategies. The IAU 2000A nutation model is complemented by the new precession $\mathrm{P} 03$ development of Capitaine et al. (2003) that was recently adopted by the IAU (Resolution 1, IAU 26th General Assembly, Prague, August 2006).

The rigid Earth nutation theories available in the literature (SMART97 of Bretagnon et al. 1998; RDAN97 of Roosbeek \& Dehant 1998; REN 2000 of Souchay et al. 1999) are all truncated at $0.1 \mu$ as or less. Nevertheless, differences between the observed precession and nutation derived from VLBI measurements and IAU 2000A are at the level of 50 microarc second per year $(\mu \mathrm{as} / \mathrm{yr})$ and $100 \mu$ as, respectively. These differences reflect some influences of the observational strategy as well as geophysical processes that still need to be addressed (see, e.g., Dehant et al. 2003) or high order contributions not considered before. If one wants to further study the nutation residuals and interpret them in terms of new geophysical phenomena, it is of very high priority to consider all the theoretical phenomena that can provide contributions. Our work must be placed in that context.

We examine the contribution to the transfer function for nutations of a real Earth with an elastic mantle and a liquid core, related to the existence of Poisson terms in the forcing. These terms stem from the approximation for short timescale of the long term variations arising from planetary perturbations. In Sect. 2, we provide the basic angular momentum balance equations for computing the Earth wobbles at all time scales. The solutions are given in Sect. 3 for the whole Earth wobble and for the core differential wobble. A numerical evaluation along with a discussion are given in Sect. 4.

\section{The Earth's variable rotation}

We consider an Earth model consisting of an elastic mantle and a fluid core (other refinements like a solid inner core would have negligible effects). The external gravitational potential is due to the influence of the moon, the sun and, to a minor extent, of the planets. We define the potential of spherical harmonic order $l$ and degree $m$ at any point within the Earth at geocentric distance $r$, colatitude $\theta$, east longitude $\lambda$ by

$U_{l m}(r, \theta, \lambda)=-\left(\frac{r}{a}\right)^{l} \operatorname{Re}\left(V_{l m} Y_{l m}\right)$ 
where $a$ is the Earth mean radius, and $Y_{l m}=$ $P_{l m}(\cos \theta) \exp (-\mathrm{i} m \lambda) \quad\left(P_{l m}\right.$ being the associated Legendre functions). In our convention, the force is minus the gradient of the potential. The degree 2 potential is the sum of zonal $(l=2$, $m=0)$, tesseral $(l=2, m=1)$ and sectorial $(l=2, m=2)$ components involving

$$
\begin{aligned}
& V_{20}=\frac{G M a^{2}}{d^{3}} \frac{3 \sin ^{2} \beta-1}{2}, \\
& V_{21}=V_{21}^{\mathrm{re}}+\mathrm{i} V_{21}^{\mathrm{im}}=\frac{G M a^{2}}{d^{3}} \sin \beta \cos \beta \mathrm{e}^{\mathrm{i} \Lambda}, \\
& V_{22}=V_{22}^{\mathrm{re}}+\mathrm{i} V_{22}^{\mathrm{im}}=\frac{G M a^{2}}{4 d^{3}} \cos ^{2} \beta \mathrm{e}^{2 \mathrm{i} \Lambda},
\end{aligned}
$$

where $\Omega$ is the mean Earth's rotation rate, $a$ is the Earth radius, $\beta$ and $\Lambda$ are the latitude and longitude of the positions of the perturbing celestial bodies referred to a terrestrial frame with its $x$ and $y$ axes along the equatorial principal axes of inertia, $M$ and $d$ are the mass and the geocentric distance of the perturbing body, respectively, and $G$ is the universal constant of gravitation. The apparent motion of the perturbing bodies determines the frequency spectrum of the $V_{l m}$. The zonal part of the degree 2 potential consists of low frequencies $(\ll \Omega)$. The tesseral part is in the retrograde diurnal band with frequencies $\sigma$ centered at $-\Omega$, and the sectorial part in the retrograde semidiurnal band. Only the zonal and tesseral parts of the potential will be relevant for our purpose.

The angular momentum balance equations for the whole Earth and the liquid core relate the angular velocity vectors of the whole Earth, $\omega$, and of the liquid core, $\omega_{\mathrm{f}}$, to the potential. In an Earth-fixed frame of reference, the equations for the equatorial component $\omega=\omega_{1}+\mathrm{i} \omega_{2}$ are (see, e.g., Sasao et al. 1980; Hinderer et al. 1987):

$$
\begin{aligned}
& A\left[1+\alpha \frac{k}{\kappa}\right] \frac{\mathrm{d} \omega}{\mathrm{d} t}+\left[A_{\mathrm{f}}+A \alpha \frac{\bar{k}_{1}}{\kappa}\right] \frac{\mathrm{d} \omega_{\mathrm{f}}}{\mathrm{d} t} \\
& -\mathrm{i} \Omega A \alpha\left[1-\frac{k}{\kappa}\right] \omega+\mathrm{i} \Omega\left[A_{\mathrm{f}}+A \alpha \frac{\bar{k}_{1}}{\kappa}\right] \omega_{\mathrm{f}}= \\
& -3 \mathrm{i} \frac{A \alpha}{a^{2}}\left\{V_{21}-\frac{k}{\kappa}\left[-\frac{\mathrm{i}}{\Omega} \frac{\mathrm{d} V_{21}}{\mathrm{~d} t}+V_{21}\right]\right\}, \\
& {\left[1+\frac{q_{0}}{2} h_{\mathrm{f}}\right] \frac{\mathrm{d} \omega}{\mathrm{d} t}+\left[1+\frac{q_{0}}{2} \bar{h}_{1 \mathrm{f}}\right] \frac{\mathrm{d} \omega_{\mathrm{f}}}{\mathrm{d} t}+\mathrm{i} \Omega\left(1+\alpha_{\mathrm{f}}\right) \omega_{\mathrm{f}}=} \\
& \frac{3}{a^{2} \Omega} \frac{q_{0}}{2} h_{\mathrm{f}} \frac{\mathrm{d} V_{21}}{\mathrm{~d} t} .
\end{aligned}
$$

The axial component of the angular velocity is governed by the equation:

$$
\begin{gathered}
C\left[1+\frac{4}{3} \frac{\alpha k}{\kappa}\right] \frac{\mathrm{d} \omega_{3}}{\mathrm{~d} t}+C_{\mathrm{f}}\left[1-\frac{4}{3} \frac{\alpha k_{1} C}{\kappa C_{\mathrm{f}}}\right] \frac{\mathrm{d} \omega_{3, \mathrm{f}}}{\mathrm{d} t}=\frac{2 k \alpha C}{\kappa a^{2} \Omega} \frac{\mathrm{d} V_{20}}{\mathrm{~d} t} \\
C_{\mathrm{f}}\left[1+\frac{2}{3} q_{0} h_{\mathrm{f}}\right] \frac{\mathrm{d} \omega_{3}}{\mathrm{~d} t}+C_{\mathrm{f}}\left[1-\frac{2}{3} q_{0} h_{1 \mathrm{f}}\right] \frac{\mathrm{d} \omega_{3, \mathrm{f}}}{\mathrm{d} t}=\frac{q_{0} h_{\mathrm{f}} C_{\mathrm{f}}}{a^{2} \Omega} \frac{\mathrm{d} V_{20}}{\mathrm{~d} t}
\end{gathered}
$$

In the above relations,

$V_{21}$ is the tesseral part of the potential,

$V_{20}$ is the zonal part of the potential,

$A, A_{\mathrm{f}}$, and $A_{\mathrm{m}}$ and $C, C_{\mathrm{f}}$, and $C_{\mathrm{m}}$ are the principal moments of inertia of the whole Earth, of the liquid core, and of the mantle, for the equatorial and $z$-axis (principal) mass repartition $\left(A=8 \times 10^{37} \mathrm{~kg} \mathrm{~m}^{-3}, A_{\mathrm{f}}=9.1 \times 10^{36} \mathrm{~kg} \mathrm{~m}^{-3}\right.$, and $\left.A_{\mathrm{m}}=7 \times 10^{37} \mathrm{~kg} \mathrm{~m}^{-3}\right)$,

$\alpha=\frac{C-A}{A}=\frac{1}{305}$ and $\alpha_{\mathrm{f}}=\frac{C_{\mathrm{f}}-A_{\mathrm{f}}}{A_{\mathrm{f}}}=\frac{1}{393}$ are the dynamical flattenings of the whole Earth and of the liquid core,

$k$ and $k_{1}$ are Love numbers expressing the mass redistribution potential at the surface of the Earth induced by a potential change of the whole Earth and of the liquid core respectively, $\left(k=0.3, k_{1}=0.06\right)$,

$\bar{k}_{1}$ is the Love number expressing the mass redistribution potential at the surface of the Earth induced by a pressure at the core-mantle boundary $(\mathrm{CMB})\left(\bar{k}_{1}=0.06\right)$,

$h_{\mathrm{f}}$ is the Love number expressing the deformation of the CMB induced by a volumic potential evaluated at the surface $\left(h_{\mathrm{f}}=\right.$ 1.14),

$h_{1 \mathrm{f}}$ is the Love number expressing the deformation of the CMB caused by a volumic potential evaluated at the $\mathrm{CMB}\left(h_{1 \mathrm{f}}=\right.$ $0.35)$,

$\bar{h}_{1 \mathrm{f}}$ is the Love number expressing the deformation of the CMB caused by an inertial pressure on the CMB $\left(\bar{h}_{1 \mathrm{f}}=0.35\right)$, $\kappa$ is the fluid Love number $\left(\kappa=3 \frac{\alpha A G}{\Omega^{2} a^{5}}=0.934\right)$, and $q_{0}$ is defined as $\frac{\Omega^{2} a_{\mathrm{eq}}^{3}}{G M_{\mathrm{E}}}=\frac{1}{289}$ where $M_{\mathrm{E}}$ is the Earth's mass.

The numerical values and the defining formula of the above constants have been taken from Dehant et al. (1993) and Greff-Lefftz et al. (2000) based on the PREM seismic model of Dziewonski et al. (1981).

The relations between our notations and the notation used in Sasao et al. (1980) or Mathews et al. (2002) is provided in Table 1. Notice that in the literature, one usually writes $\omega=$ $\Omega m=\Omega\left(m_{1}+\mathrm{i} m_{2}\right)$ and considers the above equations for $m$ only. Here, the quantity $\omega$ will be referred to as the wobble. Moreover, the wobble admittance for a given forcing frequency, i.e., the frequency-dependent expression linking the harmonic coefficients of the potential to the wobble, will be referred to as the transfer function, as in Dehant et al. (1993) or Dehant et al. (2005). Wahr (1981) or Mathews et al. (2002) called transfer function the ratio between the wobble for a non-rigid Earth and the wobble for a rigid Earth, which is simply the transfer function defined in our fashion divided by the corresponding wobble admittance for a rigid Earth (let $3 \alpha / a^{2}(\alpha \Omega-\sigma)$ for the forcing frequency $\sigma$ ).

\section{Solutions of the equations}

\subsection{Solutions for the wobble}

We now consider that the external potential contains contributions of the form $t \times \mathrm{e}^{\mathrm{i} \sigma t}$, the so-called Poisson terms. The potential can therefore be written:

$$
V_{21}=V_{21,0}+V_{21,1} t+V_{21,2} t^{2}+\sum_{n}\left(V_{21, n, 0}+V_{21, n, 1} t\right) \mathrm{e}^{\mathrm{i} \sigma_{n} t}
$$

where the amplitudes $V_{21, n, 0}$ and $V_{21, n, 1}$ are both related to the spectral component of frequency $\sigma_{n}$. Moreover, we assume that the solutions of Eqs. (5), (6) can be written as follows:

$$
\begin{aligned}
& \omega=\omega_{0}+\omega_{1} t+\omega_{2} t^{2}+\sum_{n}\left(\omega_{n, 0}+\omega_{n, 1} t\right) \mathrm{e}^{\mathrm{i} \sigma_{n} t} \\
& \omega_{\mathrm{f}}=\omega_{\mathrm{f}, 0}+\omega_{\mathrm{f}, 1} t+\omega_{\mathrm{f}, 2} t^{2}+\sum_{n}\left(\omega_{\mathrm{f}, n, 0}+\omega_{\mathrm{f}, n, 1} t\right) \mathrm{e}^{\mathrm{i} \sigma_{n} t}
\end{aligned}
$$


Table 1. Comparison between the notations concerning the compliances, Love numbers and frequencies used in the papers mentioned.

\begin{tabular}{ccc}
\hline \hline $\begin{array}{c}\text { Sasao et al. (1980)/Mathews et al. (2002) } \\
\text { Compliances/frequencies }\end{array}$ & Numerical values & This paper \\
Love numbers/frequencies \\
\hline$\kappa$ & $1.05 \times 10^{-3}$ & $\frac{k}{\kappa} \alpha$ \\
$\beta$ & $6 \times 10^{-4}$ & $\frac{q_{0}}{2} \bar{h}_{1 \mathrm{f}}$ \\
$\xi$ & $2 \times 10^{-4}$ & $\alpha \frac{\bar{k}_{1}}{\kappa}$ \\
$\gamma$ & $2 \times 10^{-3}$ & $\frac{q_{0}}{2} h_{\mathrm{f}}$ \\
$e$ & $3 \times 10^{-3}$ & $\alpha$ \\
$e_{\mathrm{f}}$ & $2 \times 10^{-3}$ & $\alpha_{\mathrm{f}}$ \\
$n_{0}$ & $-1.594 \times 10^{-7} \mathrm{rad} \mathrm{s}^{-1}$ & $\sigma_{\mathrm{FCN}}^{\prime}$ \\
$\sigma_{1}$ & $0.181 \times 10^{-6} \mathrm{rad} \mathrm{s}^{-1}$ & $\sigma_{\mathrm{CW}}$ \\
$n$ & & $\sigma$ \\
\hline
\end{tabular}

We then substitute expressions (9)-(11) in (5),(6) and solve for the coefficients appearing in (10),(11). The complete set of 10 equations is given in the Appendix. The resulting expression of the wobble $\omega$ is given, up to the first order in the small quantities such as $\alpha, \alpha_{\mathrm{f}}, q_{0}, \omega_{i}$, and $\omega_{\mathrm{f}, i}$, by

$$
\begin{aligned}
\omega & =\frac{3}{a^{2} \Omega}\left[V_{21,0}-\frac{\mathrm{i}}{\sigma_{\mathrm{CW}}} V_{21,1}-\frac{2}{\sigma_{\mathrm{CW}}^{2}} V_{21,2}\right] \\
& +\frac{3}{a^{2} \Omega}\left[V_{21,1}-\frac{2 \mathrm{i}}{\sigma_{\mathrm{CW}}} V_{21,2}\right] t+\frac{3}{a^{2} \Omega} V_{21,2} t^{2} \\
& +\sum_{n}\left[T\left(\sigma_{n}\right)\left(V_{21, n, 0}+V_{21, n, 1} t\right)+\mathrm{i} \Delta T\left(\sigma_{n}\right) V_{21, n, 1}\right] \mathrm{e}^{\mathrm{i} \sigma_{n} t}
\end{aligned}
$$

and the solution for the core wobble $\omega_{\mathrm{f}}$ is:

$$
\begin{aligned}
\omega_{\mathrm{f}} & =\frac{3}{a^{2} \Omega^{2}}\left[\mathrm{i} V_{21,1}+\frac{2}{\sigma_{\mathrm{CW}}} V_{21,2}\right]+2 \frac{3 \mathrm{i}}{a^{2} \Omega^{2}} V_{21,2} t \\
& +\sum_{n}\left[T_{\mathrm{f}}\left(\sigma_{n}\right)\left(V_{21, n, 0}+V_{21, n, 1} t\right)+\mathrm{i} \Delta T_{\mathrm{f}}\left(\sigma_{n}\right) V_{21, n, 1}\right] \mathrm{e}^{\mathrm{i} \sigma_{n} t} .
\end{aligned}
$$

where $T$ and $T_{\mathrm{f}}$ are the transfer functions given by

$$
\begin{aligned}
& T(\sigma)=\frac{3}{a^{2} \Omega} \\
& \times\left[\frac{\alpha A k-\frac{q_{0}}{2} h_{\mathrm{f}} A_{\mathrm{f}} \kappa}{\kappa A_{\mathrm{m}}}+\frac{\left(\alpha-\frac{q_{0}}{2} h_{\mathrm{f}}\right) \sigma_{\mathrm{FCN}}^{\prime} A_{\mathrm{f}}}{A_{\mathrm{m}}\left(\sigma-\sigma_{\mathrm{FCN}}\right)}-\frac{\sigma_{\mathrm{CW}}}{\sigma-\sigma_{\mathrm{CW}}}\right], \\
& T_{\mathrm{f}}(\sigma)=\frac{3}{a^{2} \Omega} \\
& \times\left[-\frac{\left(\alpha k-\kappa \frac{q_{0}}{2} h_{\mathrm{f}}\right) A}{\kappa A_{\mathrm{m}}}+\frac{\left(\alpha-\frac{q_{0}}{2} h_{\mathrm{f}}\right) \Omega A}{A_{\mathrm{m}}\left(\sigma-\sigma_{\mathrm{FCN}}\right)}+\frac{\sigma_{\mathrm{CW}}^{2}}{\Omega\left(\sigma-\sigma_{\mathrm{CW}}\right)}\right],
\end{aligned}
$$

and $\Delta T$ and $\Delta T_{\mathrm{f}}$ are additional terms to the transfer functions due to the presence of the Poisson terms in the potential and are expressed by

$$
\begin{aligned}
& \Delta T(\sigma)=\frac{3}{a^{2} \Omega}\left[\frac{-\sigma_{\mathrm{CW}}}{\left(\sigma-\sigma_{\mathrm{CW}}\right)^{2}}+\frac{\left(\alpha-\frac{q_{0}}{2} h_{\mathrm{f}}\right) A_{\mathrm{f}} \sigma_{\mathrm{FCN}}^{\prime}}{A_{\mathrm{m}}\left(\sigma-\sigma_{\mathrm{FCN}}\right)^{2}}\right] \\
& \Delta T_{\mathrm{f}}(\sigma)=\frac{3}{a^{2} \Omega}\left[\frac{\sigma_{\mathrm{CW}}^{2}}{\Omega\left(\sigma-\sigma_{\mathrm{CW}}\right)^{2}}+\frac{\left(\alpha-\frac{q_{0}}{2} h_{\mathrm{f}}\right) A \Omega}{A_{\mathrm{m}}\left(\sigma-\sigma_{\mathrm{FCN}}\right)^{2}}\right] .
\end{aligned}
$$

In the above expressions,

$\sigma_{\mathrm{FCN}}=-\Omega\left[1+\frac{A}{A_{\mathrm{m}}}\left(\alpha_{\mathrm{f}}-\frac{q_{0}}{2} \bar{h}_{1 \mathrm{f}}\right)\right]$

is the frequency of the nearly diurnal free wobble (NDFW) associated with the free core nutation (FCN), and expressed in the terrestrial frame, $\sigma_{\mathrm{FCN}}^{\prime}=\sigma_{\mathrm{FCN}}+\Omega$ is its counterpart in the celestial reference frame, and

$\sigma_{\mathrm{CW}}=\frac{A \Omega \alpha}{A_{\mathrm{m}}}\left(1-\frac{k}{\kappa}\right)$

is the Chandler wobble (CW) frequency in the terrestrial frame.

It appears in Eq. (12) that the polynomial part of the wobble is only excited by the $V_{21, j}, j=0,1,2$, i.e., the polynomial part of the potential of the tesseral potential. Similarly, the harmonic part (both Fourier and Poisson terms) of the wobble is excited exclusively by the $V_{21, n, j}, j=0,1$, i.e., the Fourier and Poisson terms of the tesseral potential. Moreover, the solution for the wobble given by Eq. (12) contains two terms proportional to $V_{21, n, 1}$ and inversely proportional to $\left(\sigma-\sigma_{\mathrm{FCN}}\right)^{2}$ and to $\left(\sigma-\sigma_{\mathrm{CW}}\right)^{2}$, in the part of the solution that has no Poisson term. These term are new with respect to the corresponding relation between the wobble and the potential given in Dehant et al. (1993), relevant to a potential without Poisson terms.

Concerning the whole Earth's wobble, it must be noted that the additional transfer function would also exist if there would be no fluid core. There is indeed a contribution only depending upon the Chandler wobble frequency. In that case, one should take the Chandler wobble frequency for an elastic Earth without a fluid core obtained by replacing $A_{\mathrm{m}}$ by $A$ in Eq. (19). The existence of the new terms in the transfer function indicates that if one finds energy near the normal mode frequencies in the Poisson terms of the forcing, the normal modes of the Earth may also be excited.

\subsection{Low frequency polar motion}

The solution for the long-term wobble is obtained by considering only small frequencies (in the rotating frame) and trends in (12) and (13). In that case, $\sigma-\sigma_{\mathrm{FCN}}$ reduces to $\Omega$. The additional term with respect to the classical expression of Dehant et al. (1993) is small as it is proportional to $\sigma_{\mathrm{FCN}}^{\prime}$ and to $\left(\alpha-\frac{q_{0}}{2} h_{\mathrm{f}}\right) A_{\mathrm{f}}$ (both at the level of $10^{-3}$ ), as well as to $V_{21, n, 1}$, which is smaller than 
$3 \times 10^{-3} V_{21, n, 0}$. The effect of the additional term on the polar motion is therefore negligible. The same remarks apply for the core wobble.

\subsection{Nutation and precession}

The nutation angles $\Delta \psi$ and $\Delta \epsilon$ describing the motion of the Earth's figure axis in space are kinematically related to the wobble by

$\Delta \dot{\epsilon}-\mathrm{i} \Delta \dot{\psi} \sin \epsilon=-\omega \mathrm{e}^{\mathrm{i} \Omega t}$.

To compute the nutations, one must take the transfer function for the wobble in the retrograde diurnal frequency band. The frequency $\sigma$, reckoned in the Earth-fixed reference frame, is equal to $\sigma^{\prime}-\Omega$ where $\sigma^{\prime}$ is the frequency in space.

The precessional motion $\omega_{\text {prec }}$ corresponds to a terrestrial frequency $\sigma=-\Omega$ in the periodic (Fourier and Poisson) part of the wobble given in Eqs. (12) and (13). The wobble denoted by $\omega_{\text {prec }}$ has the final form:

$$
\begin{aligned}
\omega_{\text {prec }} \mathrm{e}^{\mathrm{i} \Omega t}= & \frac{3 \alpha}{a^{2} \Omega}\left(V_{21, n, 0}+V_{21, n, 1} t\right) \\
& +\frac{3 \mathrm{i}}{a^{2} \Omega}\left[\frac{A_{\mathrm{f}}\left(\alpha-\frac{q_{0}}{2} h_{\mathrm{f}}\right)}{A_{\mathrm{m}} \sigma_{\mathrm{FCN}}^{\prime}}-\frac{\sigma_{\mathrm{CW}}}{\Omega^{2}}\right] V_{21, n, 1}
\end{aligned}
$$

where we have replaced $T(\sigma)$ by $T(-\Omega)$ and $\Delta T(\sigma)$ by $\Delta T(-\Omega)$ in Eq. (12) and taken only the first order in the small quantities. We can observe in the above expression that there is an additional term of the form:

$$
\frac{3 \mathrm{i}}{a^{2} \Omega}\left[\frac{A_{\mathrm{f}}\left(\alpha-\frac{q_{0}}{2} h_{\mathrm{f}}\right)}{A_{\mathrm{m}} \sigma_{\mathrm{FCN}}^{\prime}}-\frac{\sigma_{\mathrm{CW}}}{\Omega^{2}}\right] V_{21, n, 1}
$$

which is due to the presence of a liquid core and to the Poisson term in the tidal potential at the tide $K_{1}$ corresponding to precession. This additional part contains two terms, the first one being 20 times larger than the second one.

The core precession $\omega_{\mathrm{f}, \text { prec }}$ is obtained by replacing $\sigma$ by $-\Omega$ in (13):

$$
\begin{aligned}
& \omega_{\mathrm{f}, \mathrm{prec}} \mathrm{e}^{\mathrm{i} \Omega t}= \\
& -\frac{3}{a^{2} \Omega}\left[\frac{A\left(\alpha k-\kappa \frac{q_{0}}{2} h_{\mathrm{f}}\right)}{\kappa A_{\mathrm{m}}}+\frac{A \Omega\left(\alpha-\frac{q_{0}}{2} h_{\mathrm{f}}\right)}{\sigma_{\mathrm{FCN}}^{\prime} A_{\mathrm{m}}}\right]\left(V_{21, n, 0}+V_{21, n, 1} t\right) \\
& +\frac{3 \mathrm{i}}{a^{2} \Omega} \frac{A \Omega\left(\alpha-\frac{q_{0}}{2} h_{\mathrm{f}}\right)}{\sigma_{\mathrm{FCN}}^{\prime 2} A_{\mathrm{m}}} V_{21, n, 1}
\end{aligned}
$$

Another way to obtain the precession is to consider that we have a very long period in space in the equation and to take a Taylor expansion around the present time considering a small frequency. We have verified that this provides us with exactly the same solution.

\subsection{Axial component}

As for the wobble, we consider

$$
\begin{aligned}
\omega_{3} & =\omega_{3,0}+\omega_{3,1} t+\omega_{3,2} t^{2}+\left(\omega_{3, n, 0}+\omega_{3, n, 1} t\right) \mathrm{e}^{\mathrm{i} \sigma_{n} t}, \\
\omega_{3, \mathrm{f}} & =\omega_{3, \mathrm{f}, 0}+\omega_{3, \mathrm{f}, 1} t+\omega_{3, \mathrm{f}, 2} t^{2}+\left(\omega_{3, \mathrm{f}, n, 0}+\omega_{3, \mathrm{f}, n, 1} t\right) \mathrm{e}^{\mathrm{i} \sigma_{n} t},
\end{aligned}
$$

in response to a zonal tidal potential of the form

$V_{20}=V_{20,0}+V_{20,1} t+V_{20,2} t^{2}+\left(V_{20, n, 0}+V_{20, n, 1} t\right) \mathrm{e}^{\mathrm{i} \sigma_{n} t}$.

The solution of the third component of the Liouville equation then takes the form:

$$
\begin{aligned}
\omega_{3}= & \frac{2}{a^{2} \Omega} \frac{\alpha k C-\frac{q_{0}}{2} h_{\mathrm{f}} \kappa C_{\mathrm{f}}}{\kappa A_{\mathrm{m}}} \\
& \times\left[V_{20,1} t+V_{20,2} t^{2}+\left(V_{20, n, 0}+V_{20, n, 1} t\right) \mathrm{e}^{\mathrm{i} \sigma_{n} t}\right], \\
\omega_{3, \mathrm{f}}= & -\omega_{3} .
\end{aligned}
$$

The zonal tidal potential induces deformations which, in turn, induce length-of-day variations. These variations are seen in the third component of the instantaneous rotation vector. They have been computed by, e.g., Defraigne \& Smits (1999). We note that our Poisson terms do not change the transfer function in this case but only the Poisson terms in the tidal potential itself must be considered. As the length-of-day changes induced by tides are already small (of the order of $10^{-3} \mathrm{~s}$ on UT1 as given in the above mentioned paper) and as the Poisson terms in the tidal potential are very small $\left(V_{20, n, 1}\right.$ are at the level of $3 \times 10^{-3}$ of $V_{20, n, 0}$ for instance), there is no visible contribution to length-ofday variations.

\section{Numerical evaluation of the new contributions to the precession-nutation and concluding remarks}

A numerical evaluation of the above terms can be done using a semi-analytical development of the tide generating potential (TGP) including Poisson terms coming from secular variations of lunisolar and planetary orbital parameters. We use the RATGP95 (Roosbeek 1998). Even though the additional terms appearing in the transfer function $\Delta T(\sigma)$, proportional to $\left(\sigma-\sigma_{\mathrm{N}}\right)^{-2}$ where the $\sigma_{\mathrm{N}}$ represent the resonance frequencies, are several orders of magnitude above the other well-known terms proportional to $\left(\sigma-\sigma_{\mathrm{N}}\right)^{-1}$, the resulting effect on the nutation angles is expected to be considerably lowered by the fact that the Poisson terms in the TGP (the $V_{21, n, 1}$ ) are small. For instance, one has for the 18.6-yr term, $V_{21, n, 1} / V_{21, n, 0} \sim 10^{-13}$ in SI units, and $\Delta T / T \sim 10^{6}$, so that the wobble is expected to be $10^{-7}$ of the leading term, i.e., around $1 \mu$ as. Results of the computation for the nutation angles $\Delta \psi$ and $\Delta \epsilon$ are expressed as series of periodic terms, in which the phases are linear combination of Delaunay's fundamental arguments $\left(l, l^{\prime}, F, D, \Omega\right)$. Resulting amplitudes of the nutation angles larger than $0.5 \mu$ as are reported in Table 2. The contribution to the long term nutation reaches $6 \mu$ as on the 10468 -yr term. Except for the 18.6-yr term which shows up at $1 \mu$ as, the rest of the long term nutation terms are negligible.

The contribution to the precession and obliquity rates is retrieved from Eqs. (21) and (22). The obliquity rate has two terms. The first term contains core effects and is found at $84.72 \mu \mathrm{as} / \mathrm{cy}$. The second term contributes marginally, accounting for $2.81 \mu \mathrm{as} / \mathrm{cy}$, yielding a net effect of $87.53 \mu \mathrm{as} / \mathrm{cy}$.

The quantification of the wobble/nutation related to Poisson terms and higher polynomial terms might be calculated from a numerical integration that contains both the translational equations of motion ( $N$-body problem) and rotational equations of the Earth in the solar system.

This comparison highlights, however, a difficulty related to the representation of the secular motion on short periods of time by Fourier series and Poisson terms. Bretagnon et al. (1997) first identified this problem during the elaboration of their rigid 
Table 2. Contribution of the second-order terms to the nutation.

\begin{tabular}{rrrrrrrrr}
\hline \hline$l$ & $l^{\prime}$ & $F$ & $D$ & $\Omega$ & $\begin{array}{r}\text { Period } \\
\text { days }\end{array}$ & $\begin{array}{r}\Delta \psi \\
\mu \text { as }\end{array}$ & $\begin{array}{r}\Delta \epsilon \\
\mu \text { as }\end{array}$ \\
\hline 0 & -1 & 0 & 0 & 0 & -365.26 & -0.4 & 0.2 \\
0 & 0 & 0 & 0 & 1 & -6798.38 & 0.5 & -0.2 \\
0 & 2 & -2 & 2 & -2 & -3823298.12 & 5.9 & -2.3 \\
0 & 0 & 0 & 0 & -1 & 6798.38 & -0.7 & 0.3 \\
\hline
\end{tabular}

Earth model SMART97. Unlike the other scientists working on rigid Earth nutation theory, they solved that problem by considering developments for the long period contributions around the present-day date. One of the Doodson fundamental arguments, the mean tropic longitude of perihelion $p_{\mathrm{s}}$, is for instance a long-period term that is considered in tidal theories and that is not taken into account in the analytical planetary theories (e.g., VSOP87, Bretagnon \& Francou 1988). Consequently, it is artificially introduced in the tidal potential developments. For example, taking into account $p_{\mathrm{s}}$ as a fundamental argument in the tide generating potential leads to a generation of two nutation periods separated by this argument. This is for instance the case for the principal 18.6 year nutation of which the amplitude shows a departure of 6 mas in the representation of the Earth's nutation used by Bretagnon et al. (1997) with respect to the other representations of Souchay et al. (1999) and Roosbeek $\&$ Dehant (1998). Actually, the argument $p_{\mathrm{s}}$ should be expressed as a polynomial of time when its behavior is considered around the present date. In this case, it appears in wobble or nutation as in-phase and out-of-phase components. These in-phase and outof-phase components introduce supplemental crossing terms in the calculus of $\Delta T(\sigma) V_{21, n, 1}$. Consequently, a $N$-body approach of the problem will provide various additional terms due to long periods and secular trends related to planetary perturbations that prevent comparison and identification of the impact of Poisson and higher terms on the rotational motion of the Earth.

Acknowledgements. The research was carried out in the Section Heure, Rotation de la Terre et Géodésie Spatiale of the Royal Observatory of Belgium and received financial support from the Descartes Prize Allowance (M.F.), for which we express our sincere appreciation. N.R. acknowledges the support of the Prodex/ESA contract C90253. We are grateful to O. de Viron who so kindly spent his time discussing the issues related to this work. We also thank an anonymous referee for the valuable suggestions to improve this manuscript.

\section{Appendix A: The algebraic equations accounting for the Poisson terms}

The substitution of the expressions (10) and (11) for $\omega$ and $\omega_{\mathrm{f}}$, as well as (9) for the external potential, into Eqs. (5) and (6), gives us the following set of ten algebraic equations:

$$
\begin{gathered}
\text { [1] } A\left\{\sigma+\mathrm{i} \alpha\left[-\Omega+\frac{k}{\kappa}(\sigma+\Omega)\right]\right\} \omega_{n, 1} \\
+\mathrm{i}(\sigma+\Omega)\left[A_{\mathrm{f}}+\frac{\alpha A \bar{k}_{1}}{\kappa}\right] \omega_{\mathrm{f}, n, 1}= \\
\frac{3 \mathrm{i} \alpha A}{\Omega a^{2}}\left[-\Omega+\frac{k}{\kappa}(\sigma+\Omega)\right] V_{21, n, 1}
\end{gathered}
$$

$$
\begin{aligned}
& \text { [2] } \mathrm{i} A\left[(\sigma-\Omega \alpha)+\frac{\alpha k}{\kappa}(\sigma+\Omega)\right] \omega_{n, 0}+A\left[1+\frac{\alpha k}{\kappa}\right] \omega_{n, 1} \\
& +\mathrm{i}(\sigma+\Omega)\left[A_{\mathrm{f}}+\frac{\alpha A \bar{k}_{1}}{\kappa}\right] \omega_{\mathrm{f}, n, 0}+\left[A_{\mathrm{f}}+\frac{\alpha A \bar{k}_{1}}{\kappa}\right] \omega_{\mathrm{f}, n, 1}= \\
& \quad \frac{3 \mathrm{i} \alpha A}{\Omega a^{2}}\left[-\Omega+\frac{k}{\kappa}(\sigma+\Omega)\right] V_{21, n, 0}+\frac{3 \alpha A k}{\Omega a^{2} \kappa} V_{21, n, 1} \\
& \text { [3] } \mathrm{i} \Omega \alpha A\left[-1+\frac{k}{\kappa}\right] \omega_{0}+A\left[1+\frac{\alpha k}{\kappa}\right] \omega_{1} \\
& +\mathrm{i} \Omega\left[A_{\mathrm{f}}+\frac{\alpha A \bar{k}_{1}}{\kappa}\right] \omega_{\mathrm{f}, 0}+\left[A_{\mathrm{f}}+\frac{\alpha A \bar{k}_{1}}{\kappa}\right] \omega_{\mathrm{f}, 1}= \\
& \frac{3 \mathrm{i} \alpha A}{a^{2}}\left[-1+\frac{k}{\kappa}\right] V_{21,0}+\frac{3 \alpha A k}{\Omega a^{2} \kappa} V_{21,1}
\end{aligned}
$$

[4] $\mathrm{i} \Omega \alpha A\left[-1+\frac{k}{\kappa}\right] \omega_{1}+2 A\left[1+\frac{\alpha k}{\kappa}\right] \omega_{2}$

$+\mathrm{i} \Omega\left[A_{\mathrm{f}}+\frac{\alpha A \bar{k}_{1}}{\kappa}\right] \omega_{\mathrm{f}, 1}+2\left[A_{\mathrm{f}}+\frac{\alpha A \bar{k}_{1}}{\kappa}\right] \omega_{\mathrm{f}, 2}=$

$\frac{3 \mathrm{i} \alpha A}{a^{2}}\left[-1+\frac{k}{\kappa}\right] V_{21,1}+\frac{6 \alpha A k}{\Omega a^{2} \kappa} V_{21,2}$

[5] $\mathrm{i} \Omega \alpha A\left[-1+\frac{k}{\kappa}\right] \omega_{2}+\mathrm{i} \Omega\left[A_{\mathrm{f}}+\frac{\alpha A \bar{k}_{1}}{\kappa}\right] \omega_{\mathrm{f}, 2}=$

$$
\frac{3 \mathrm{i} \alpha A}{a^{2}}\left[-1+\frac{k}{\kappa}\right] V_{21,2}
$$

[6] $\mathrm{i} A_{\mathrm{f}} \sigma\left[1-\frac{1}{2} q_{0} h_{\mathrm{f}}\right] \omega_{n, 1}$

$$
+\mathrm{i} A_{\mathrm{f}}\left[(\sigma+\Omega)+\Omega \alpha_{\mathrm{f}}+\frac{1}{2} q_{0} \bar{h}_{1 \mathrm{f}} \sigma\right] \omega_{\mathrm{f}, n, 1}=
$$

$\frac{3}{2} \frac{\mathrm{i} A_{\mathrm{f}} q_{0} h_{\mathrm{f}} \sigma}{a^{2} \Omega} V_{21, n, 1}$

[7] $\mathrm{i} A_{\mathrm{f}} \sigma\left[1+\frac{1}{2} q_{0} h_{\mathrm{f}}\right] \omega_{n, 0}+A_{\mathrm{f}}\left[1+\frac{1}{2} q_{0} h_{\mathrm{f}}\right] \omega_{n, 1}$

$+\mathrm{i} A_{\mathrm{f}}\left[(\sigma+\Omega)+\Omega \alpha_{\mathrm{f}}+\frac{1}{2} q_{0} \bar{h}_{1 \mathrm{f}} \sigma\right] \omega_{\mathrm{f}, n, 0}$

$+A_{\mathrm{f}}\left[1+\frac{1}{2} q_{0} \bar{h}_{1 \mathrm{f}}\right] \omega_{\mathrm{f}, n, 1}$

$=\frac{3}{2} \frac{\mathrm{i} A_{\mathrm{f}} q_{0} h_{\mathrm{f}} \sigma}{a^{2} \Omega} V_{21, n, 0}+\frac{3}{2} \frac{A_{\mathrm{f}} q_{0} h_{\mathrm{f}}}{a^{2} \Omega} V_{21, n, 1}$

[8] $A_{\mathrm{f}}\left[1+\frac{1}{2} q_{0} h_{\mathrm{f}}\right] \omega_{1}+\mathrm{i} \Omega A_{\mathrm{f}}\left(1+\alpha_{\mathrm{f}}\right) \omega_{\mathrm{f}, 0}$

$$
+A_{\mathrm{f}}\left[1+\frac{1}{2} q_{0} \bar{h}_{1 \mathrm{f}}\right] \omega_{\mathrm{f}, 1}=\frac{3}{2} \frac{\mathrm{i} A_{\mathrm{f}} q_{0} h_{\mathrm{f}} \sigma}{a^{2} \Omega} V_{21,1}
$$

[9] $A_{\mathrm{f}}\left(2+q_{0} h_{\mathrm{f}}\right) \omega_{2}+\mathrm{i} \Omega A_{\mathrm{f}}(1+\alpha) \omega_{\mathrm{f}, 1}$

$$
+A_{\mathrm{f}}\left(2+q_{0} \bar{h}_{1 \mathrm{f}}\right) \omega_{\mathrm{f}, 2}=3 \frac{A_{\mathrm{f}} q_{0} h_{\mathrm{f}}}{a^{2} \Omega} V_{21,2}
$$




$$
\text { [10] } \omega_{\mathrm{f}, 2}=0 \text {. }
$$

This set of equations allows us to directly obtain the expressions for $\omega_{0}, \omega_{1}, \omega_{2}, \omega_{n 0}, \omega_{n 1}, \omega_{\mathrm{f} 0}, \omega_{\mathrm{f} 1}, \omega_{\mathrm{f} 2}, \omega_{\mathrm{f} n 0}$ and $\omega_{\mathrm{f} n 1}$ in terms of the coefficients of the external potential: $V_{0}, V_{1}, V_{2}, V_{n 0}$ and $V_{n 1}$.

\section{References}

Bretagnon, P., \& Francou, G. 1988, A\&A, 202, 309

Bretagnon, P., Francou, G., Rocher, P., \& Simon, J. L.1998, A\&A, 329, 329

Capitaine, N., Wallace, P.T., \& Chapront, J. 2003, A\&A, 412, 567

Defraigne, P., \& Smits, I. 1999, Geophys. J. Int., 139, 2, 563

Dehant, V., Hinderer, J., Legros, H., \& Lefftz, M. 1993, Phys. Earth Planet. Inter. 76,259
Dehant, V., Feissel-Vernier, M., de Viron, O., et al. 2003, J. Geophys. Res., 108(B5), 10.1029

Dehant, V., de Viron, O., \& Greff-Lefftz M. 2005, A\&A, 438, 1149

Dziewonski, A.M., \& Anderson, D. L.1981, Phys. Earth Planet. Inter., 25, 297

Greff-Lefftz, M., Legros, H., \& Dehant, V. 2000, Phys. Earth Planet. Inter., 122, 187

Hinderer, J., Legros H., \& Amalvict, M. 1987, Phys. Earth Planet. Inter., 49(3-4), 213

Mathews, P. M., Herring, T. A., \& Buffett, B. A. 2002, J. Geophys. Res., 10.1029 McCarthy, D.D., \& Petit, G. 2004, Conventions 2003, IERS Technical Note, 32, Publ. Frankfurt am Main: Verlag des Bundesamts für Kartographie und Geodäsie

Roosbeek, F., \& Dehant, V. 1998, Celest. Mech., 70, 215

Sasao, T., Okubo, S., \& Saito, M. 1980, Proc. IAU Symp., 78, 165

Souchay, J., Loysel, B., Kinoshita, H., \& Folgueira, M. 1999, A\&AS, 135, 111

Wahr, J. M. 1981, Geophys. J. R. Astron. Soc., 64, 705 